\title{
Structural insights into the binding of MMP9 inhibitors
}

\author{
Arpit $\operatorname{Tandon}^{1} *$, Siddharth Sinha ${ }^{2}$
}

${ }^{1}$ Amity Institute of Biotechnology, Amity University Lucknow Campus, Gomti Nagar, Lucknow 226010, India; ${ }^{2}$ ACS BioInformatics, Biotech Park, Janki Puram, Lucknow 226021, India; Arpit Tandon: Email- arpit.tandon@ymail.com; *Corresponding author.

Received September 14, 2010; Accepted December 02, 2010; Published January 22, 2011

\begin{abstract}
:
Matrix Metalloproteinase are family of enzymes responsible for degradation of extracellular matrix. MMP9 (gelatinase B) is one of the common matrix metalloproteinase that is associated with tissue destruction in a number of disease states such as rheumatoid arthiritis, fibrotic lung disease, dilated cardiomyopathy, as well as cancer invasion and metastasis. Recent study demonstrates that increased expression of MMP9 results in augmentation of myopathy with increased inflammation and fibernecrosis. Previous studies do not provide any conclusive information related to structural specificity of MMP9 inhibitors towards its active site, but with the availability of experimental structures it is now possible to study the structural specificity of MMP9 inhibitors. In light of availability of this information, we have applied docking and molecular dynamics approach to study the binding of inhibitors to the active site of MMP9. Three categories of inhibitor consisting of sulfonamide hydroxamate, thioester, and carboxylic moieties as zinc binding groups (ZBG) were chosen in the present study. Our docking results demonstrate that thioester based zinc binding group gives favourable docking scores as compared to other two groups. Molecular Dynamics simulations further reveal that tight binding conformation for thioester group has high specificity for MMP9 active site. Our study provides valuable insights on inhibitor specificity of MMP9 which provides valuable hints for future design of potent inhibitors and drugs.
\end{abstract}

\section{Background:}

MMPs (Matrix metalloporteinases) are responsible for digestion of various structural components of extracellular matrix (ECM), such as membrane collagens, elastin, aggrecan, fibronectin and laminin [1]. In addition to their ECM substrates, MMPs also cleave other extracellular proteins. Their targets include a number of cell surface receptors, peptide growth factors, cytokines and chemokines as well as other proteases [2]. MMP proteolysis plays a central role in biological processes like embryonic development, morphogenesis, bone remodelling, homeostasis, wound repair etc. [1]. For this reason, MMPs are closely regulated in their expression and tightly controlled in their function by endogenous mechanisms such as tissue inhibitors of metalloproteinase (TIMPs). Recent study demonstrated that imbalance in this regulation results in variety of disease states such as tumor metastasis, and multiple sclerosis $[\mathbf{1}, \mathbf{3}]$.

MMPs are zinc containing endopeptidases, which belong to metzincin group of proteases. They include the M10A subfamily, the M10 family and the MA clan of metallopeptidases [4]. In humans, more than 27 different MMPs have been described which are sub-grouped into collagenases, gelatinases, stromelysins, matrilysins and membrane type MMPs [4]. Except for MMP7, MMP26 and MMP23, all MMPs have a hemopexin domain connected to the catalytic domain by a hinge or linker region. Hemopexin domain mainly affects TIMP and substrate binding while catalytic domain is where the proteolytic cleavage occurs. MMP2 (gelatinaseA) and MMP9 (gelatinaseB) are two such MMPs which are characterized by their ability to digest denatured collagens (gelatin). These MMPs have been described for their role in various pathological physiological processes in humans like inflammatory, neurodegenerative, cardiovascular and autoimmune diseases [5, 10]. MMP9 in particular has been associated with cancer cell invasion, metastasis and tumor progression [6].

MMP9 and MMP2 are distinguished among other MMPs by the presence of three head to tail cysteine rich repeats which resemble fibronectin type II repeats. This insert is required for interaction with substrates gelatin, laminin and collagen [7]. Structurally, MMP9 also contains a heavily Oglycosylated, elongated linker region between its catalytic and hemopexin domains. This O-glycosylation increases affinity for galectin-3, thus impeding metastasis formation [8]. Additionally, Role of MMP9 has been established in early tumor progression, it acts as a functional component of angiogenesis switch during multistage carcinogenesis, where MMP9 functions by triggering the release of vascular epidermal growth factor accelerating tumor growth [9]. Furthermore, increased expression of MMP9 is also observed during various neoplasias, such as tumors of colon and breasts. Therefore, from a medical perspective MMP9 is a pre-eminent target for inhibitor development, blocking MMP9 activity by more specific inhibitors can be of therapeutic benefit for patients carrying early stages of cancer.

In Nature, MMPs proteolysis is inhibited by a plasma inhibitor alpha2macroglobulin or by family of endogenous glycoproteins known as tissue inhibitors of metalloproteinases (TIMPs). Four TIMPs have been identified so far, all of which possess a conserved gene structure with 12 identically placed cysteine residues. These cysteine residues give a two domain amino terminal structure that co-ordinates bidentately to the catalytic zinc $\left(\mathrm{Zn}^{2+}\right)$ through its amino terminal moiety and carbonyl oxygen, which leads to inhibition of activity of MMPs [10]. Although TIMPs are selective inhibitors of MMPs, technical restraints prevent their development into useful drugs. However, MMPs make an attractive target for synthetic inhibitors. Most synthetic inhibitor development is based on mimicking the binding of TIMPs to the catalytic site of the enzyme. Based on this knowledge, there are two basic requirements for a molecule to be effective MMP inhibitors (MMPIs). Firstly, a functional group able to chelate the active site zinc (known as the zinc binding group). Secondly, at least one functional group capable of hydrogen bonding with the enzyme backbone. Several classes of synthetic MMP inhibitors were developed in the past. These synthetic inhibitors showed promising results during pre clinical studies on mouse models, still clinical development of MMPIs led to their subsequent failure due to various unforeseen problems. On examination of 
these failures, it was found that broad enzyme specificity of MMPIs led to musculoskeletal side effects and cell cytotoxicity [11]. These failures highlighted the need to further understand role of specific MMPs in disease pathways, their structure, and their binding to synthetic MMPIs. In recent years, crystal structures of MMP9 with its specific inhibitors have been resolved [12]. In light of this structural data, it has now become possible to evaluate the binding of potential inhibitors to MMP9. So, in current study we have applied molecular docking and molecular dynamics approach to determine structural specificity of three different ZBGs towards MMP9, the results obtained will help us understand binding of inhibitors in the active site pocket of the enzyme as well as provide clues for structure based design of potential MMP inhibitors in the future.

\section{Methodology:}

Enzyme preparation:

Crystal structure of MMP9 in complex hydroxamate inhibitor (triflouromethyl hydroxamic acid) obtained from protein data bank was used for docking studies [PDB id code: 2ow1] [12]. Water molecules and ligand were removed from the enzyme. The complex was then energy minimized in steepest descent followed by conjugate gradient method using Gromacs (version 4.0) [13]. All these minimization steps were performed using Gromos 87 force field, which is default for gromacs.

\section{Inhibitor Selection:}

The set of 54 inhibitors were selected for this study from ref 17, 18 . Selected ligands belonged to three classes of known synthetic metalloproteinase inhibitors. These three classes have hydroxamate, sulfodiimine, and carboxylate as their zinc binding groups. Chemical structures of ligands were retrieved from Pubchem compound database. In order to obtain stable ligand confirmations, they were optimized by steepest descent in Amber Force field using UCSF chimera [14].

\section{Docking Setup:}

Docking was performed using Autodock 4 and Flexidock available in SYBYL-X [15, 16]. Autodock combines energy evaluation through precalculated grids of affinity potential employing various search algorithms to find the suitable binding position for a ligand on a given protein [15]. Kollman united atom charges and polar hydrogens were added to the receptor PDB using Autodock tools [15]. All rotatable bonds in the ligands were kept free to allow for flexible docking. Binding site for ligand was chosen around Leu-187 side chain which includes all amino acid residues in the active site. Grid size was set to $60 \times 60 \times 60$ grid points (x, y and z) with spacing between grid points kept at $0.375 \AA$. The Lamarckian genetic algorithm was chosen to search for the best conformers. Standard docking protocol was applied which is based on population size of 150 randomly placed individuals, a maximum number of 250000 energy evaluations, a mutation rate of 0.02 , a crossover rate of 0.80 and an elitism value of 1 . Twenty five independent docking runs were carried out for each inhibitor and cluster tolerance was kept at $1.0 \AA$. Best docking conformers from each class of ligand were further verified using Flexidock available from SYBYL-X, each flexidock simulation was performed with 25,000 generations. Binding site was defined around $3 \AA$ region of previously docked ligand, the resulting conformations from flexidock runs were compared with autodock to establish veracity of autodock runs.

\section{Molecular Dynamics setup:}

Based on Docking results, Molecular Dynamics simulations of Active site MMP9- inhibitor complexes were carried out using Gromacs 4.0 suite of programs using gromos force field [13]. Each of the complexes was placed in centre of $90 * 90 * 90 \AA ̊$ cubic box and solvated by SPC/E water molecules [13]. The gromacs topology file for ligands was generated using the PRODRG2 server. The time constant for berendsen temperature coupling and berendsen pressure coupling were both set at 0.1 . The environment was set to $300 \mathrm{~K}$ and 1 bar. All of the complexes were energy minimized using steepest descent method. Further, a 30 ps position restraining simulation was carried out to restrict the movement of the protein in the simulation. For the long range electrostatic interactions, Particle Mesh Ewald (PME) electrostatic was used. The cut-off for coulomb interaction and Vander Waal interaction were set to $1.0 \mathrm{~nm}$ and $1.4 \mathrm{~nm}$ respectively. The LINCS algorithm was used for all bond constraints.

\section{Results and Discussion:}

Even though, several crystal structures of MMP9 in complex with other inhibitors are present, structure of truncated MMP9 in complex with hydroxamic acid inhibitor [PDB id code 2OW1] was used for docking studies. In this structure, the hydroxamic acid group demonstrates high affinity for catalytic zinc atom by co-ordinating in a bidentate manner through its hydroxyl and carbonyl groups. Overall, structure of the complex displays tight binding conformation with almost exact resemblance to structure of Pro-MMP9 isoform with slight disordering in Leu 187 side chain [12]. Moreover, the subsite conformations in this structure allow for favorable hydrophobic and electrostatic interactions with residues in main substrate binding cavity. Therefore, the structure provides optimal conformation for docking small molecules in the active site.

Docking was performed using Autodock4.0. To verify the accuracy of docking results, hydroxamate (triflouromethyl hydroxamic acid) was extracted from crystal structure in its experimental conformation. It was docked back to the corresponding binding pocket. The top ranking conformational clusters from this dock were evaluated in terms of root mean square deviation between docked position and experimentally determined position for the ligand. The low RMS $(<2 \AA)$ between the experimental and docked co-ordinates of ligand indicated energetically favorable and statistically validated docking result. Further verification of docking results was performed using flexidock module available in SYBYL-X, this was done to account for interaction of $\mathrm{Zn}$ atom present in protein with the ligand.

For docking studies, a set of 54 inhibitors were chosen from three broad classes of MMP inhibitors: (1) carboxylic acid based inhibitors (2) Sulfonamide hydroxamate based inhibitors (3) Thio ester based inhibitors $[17,18]$. This classification is based upon the nature of zinc binding group (ZBG) present. All the inhibitors were then docked into the active site of MMP9. Active site of MMP9 comprises of catalytic zinc ion and is separated into small "lower" and large "upper" subdomain. These subdomains form $\mathrm{Sn}(\mathrm{n}=1,2,3 \ldots)$ and $\mathrm{Sn}$ ' $(\mathrm{n}$ '=1, 2, 3...) substrate binding pockets, small inhibitors bind to the $\mathrm{S} 1$ ' substrate binding pocket, formed by these two subdomains [19]. S1' pocket is framed in the center of active site cleft closest in proximity to active site zinc. This pocket comprises of Asp 185 - Leu 188 and Pro 421-Tyr 423 which provide donors and acceptors for inter main chain hydrogen bonds to substrates or inhibitors. The wall of S1' cavity is formed by side chains of Leu 188, Leu 397, Val 398, His 401, and Leu 418, and the Met 422-Tyr 423 main chain. Leu 397 and Val 398 are specific to MMP9 [12]. The 25 docking conformations for each ligand were divided into separate conformational clusters according to $2 \AA$ RMSD criteria. Autodock ranks each conformational cluster by binding free energy evaluation to find the best binding mode [15]. Best ranking conformational clusters from each class of ligand were further docked using Flexidock module in SYBYL-X. Results from both autodock and flexidock show agreement with less than $1 \AA$ RMSD deviation in the conformation of docked ligands. In our results best ranking conformations demonstrated a clear propensity to bind into the S1' subsite within the active site cleft. The orientation of ZBG in all of 54 best ranking clusters was similar to the orientation of ZBG of ligand in the experimental structure. Our docking results clearly show that co-ordinate bond formation between ZBG and active site zinc is facilitated by anchoring of functional groups of ligand with residues in S1' cavity. Highest ranking conformations in 43 of the 54 docks hydrogen bond either with upper domain Leu 188-Ala 189 or lower domain Gln 402-His 411. Anchoring in the upper part of S1' subsite is supported mainly by main chain amine moeity of Leu 188, which acts as a hydrogen bond donor to functional group of ligands. This interaction determines positioning of ligand within the ' $\mathrm{S} 1$ ' subsite. It was observed that bulkier functional group moieties bind deep into the upper domain Leu 188 pocket of the subsite while smaller functional group moieties show preference for Gln 402-His 411 


\section{Bioinformation}

pocket. Apart from above mentioned residues, S1' wall residues Pro 421Tyr 423 also act as hydrogen bond acceptor in few ligand conformations. Docking results reaffirm the fact that $\mathrm{S}^{\prime}$ ' subsite is likely the most important substrate recognition point in the active site cleft.

The binding free energy evaluation by Autodock includes intermolecular energy (van der Waals energy, hydrogen bonding energy, desolvation energy, and electrostatic energy), internal energy and torsional energy. The first two items build up docking energy; the first and third items compose the binding energy. The difference in binding free energy of three different classes of inhibitors provides further insights into their binding mode with MMP9. Our docking results indicate that Thiol based ZBG Zinc Binding Group is energetically favored over carboxylic and hydroxamate Zinc Binding Groups. Analysis of docking poses of Thio ester based ZBG group suggests that while bivalent sulfur helps in co-ordinating monodentately with the catalytic zinc, with the two bulkier moieties attached to thio ester strongly interacting with $\mathrm{S}^{\prime}$ ' subsite (13 of the 18 top ranking conformations interact with both lower and upper domain S1' residues). In case of hydroxamate and carboxylic groups, only one functional group can co-ordinate with the catalytic zinc thus restricting the ability to interact with S1' subsite. Analysis of binding energies within each group demonstrates that ligands with bulkier side chains in functional groups have better binding energies than smaller side chains. It can be argued that the bulkier moieties attached to thio ester can also possibly extend into S1 and S2' subsites resulting in increased interactions between ligand and enzyme. Based on docking results, one enzyme-inhibitor conformation from each of the three groups was chosen for molecular dynamics studies (Table 1 see supplementary material). This choice was based on the optimal binding conformation obtained by inhibitor in the group. The optimal conformation was ascertained on following criteria: docking energy, binding conformation, and hydrogen bonding with active site residues. We examined the mobility of inhibitors in active site through $1 \mathrm{~ns}$ MD simulation. This was done by calculating RMSD of inhibitors atom position to MMP9 backbone for all three systems (Figure 2). A comparison of RMSD deviation and subsequent conformational changes indicate binding mobility in all three groups of inhibitor.

For the carboxylic group, in the first $250 \mathrm{ps}$ of simulation, the inhibitor aligns itself to a more vertical position subsequently loses contact with $\mathrm{S} 1$ subsite resulting in opening up of active site cleft. However, about halfway through the simulation inhibitor anchors itself to S1' subsite again. This occurs by anchoring with the side chains of Ala-189 and Leu-188 and finally closing the active site again. This conformation gradually stabilizes at about $700 \mathrm{ps}$ with slight changes in backbone structure. For the hydroxamate group, the inhibitor changes its original binding conformation and aligns itself in S1 subsite. The active site pocket achieves an open conformation while inhibitor further buries itself in $\mathrm{S} 1$ pocket. This results in increased interaction of hydroxamate moiety with His 113 and brings about slight closing of active site cleft. This conformation attains stability at around $700 \mathrm{ps}$ and remains stable for rest of the simulation. For the Thiol based ZBG group, the binding conformation of the inhibitor forces the active site to remain in a closed conformation for most of the simulation period. Still, around 300 ps the inhibitor realigns itself in the S1' subsite further closing the active site to attain very tight binding conformation, and this conformation remains stable for rest of the simulation.

All three inhibitors display varying mobility in the active site pocket as evidenced by MD simulations. A further calculation of $500 \mathrm{ps}$ reveals that the structures attain conformational stability around 1000 to $1100 \mathrm{ps}$ for all three groups. The reasons for realignment are explained by the nature S1 and S1' subsites. S1' is a deep hydrophobic pocket which acts as substrate recognition point while $\mathrm{S} 1$ forms a hydrophobic surface which accommodates amino acids from the substrates. S1 subsite acts as a channel for amide bond hydrolysis by exposing amide bonds to nucleophilic zinc at the center of active site. Carboxylic and hydroxamate group inhibitors demonstrate this by first binding to energetically favorable S1' pocket with their bulky side chains preferring deep hydrophobic pockets, however simulation reveals that polar residues (Tyr 423) push aromatic side chains of inhibitors to realign into $\mathrm{S} 1$ pocket. It is revealed that bulky Thiol based ZBG inhibitor buries deep inside active site cleft closing face of the active site, and it shows steadier anchoring within the active site pocket compared to other two groups. Thio ester based ZBG group inhibitor also interacts with Val 398, which is specific to MMP9.

\section{Conclusion:}

In our study, molecular docking and dynamics approach was applied to study the binding conformations and structural specificity of three new groups of MMP inhibitors. In three groups, carboxylic acid ZBG and sulfonamide hydroxamate ZBG demonstrate similar binding orientations with general specificity towards $\mathrm{S} 1$ ' pocket of the enzyme with sulfonamide based hydroxamate group that anchoring more steadily into the $\mathrm{S} 1$ ' pocket by having more hydrogen bonding pairs between enzyme and inhibitor. Moreover, thio-ester based ZBG displays better binding and greater specificity than the other two inhibitor groups, which have the ability to anchor to both S1 and S1' subsite and maintain a tightly closed conformation throughout the simulation period that indicate of greater specificity towards the structure of the enzyme.

Our study explores structural specificity of three groups of inhibitors towards recently available MMP9 structure and provides valuable insights into designing highly specific inhibitors towards MMP9 enzyme. In this study, thio ester ZBG compounds demonstrate promising results, and further studies could provide leads for highly selective inhibitors for MMP9 enzyme in the future.

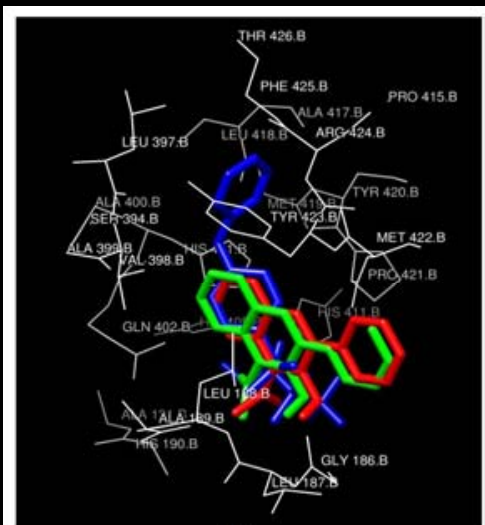

(a)

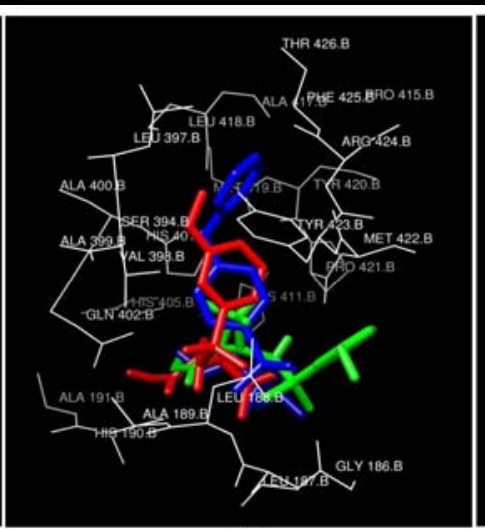

(b)

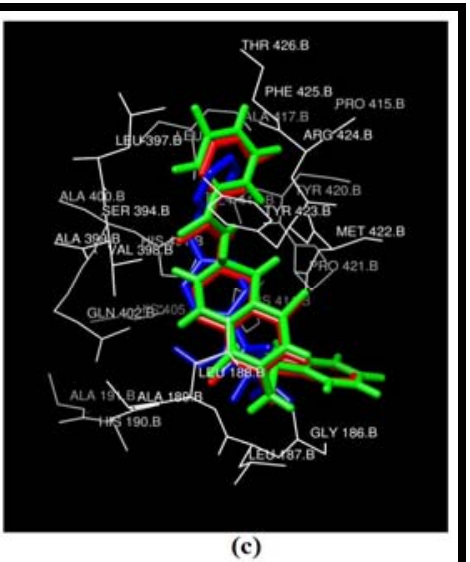

Figure 1: Docked conformations of Inhibitors in Red (Autodock) and Green (Flexidock) with respect to crystal structure ligand in blue. (a) Carboxylic acid ZBG (b) Sulfonamide Hydroxamate ZBG (c) Thio ester based ZBG 


\section{Bioinformation}

\section{Volume 5}

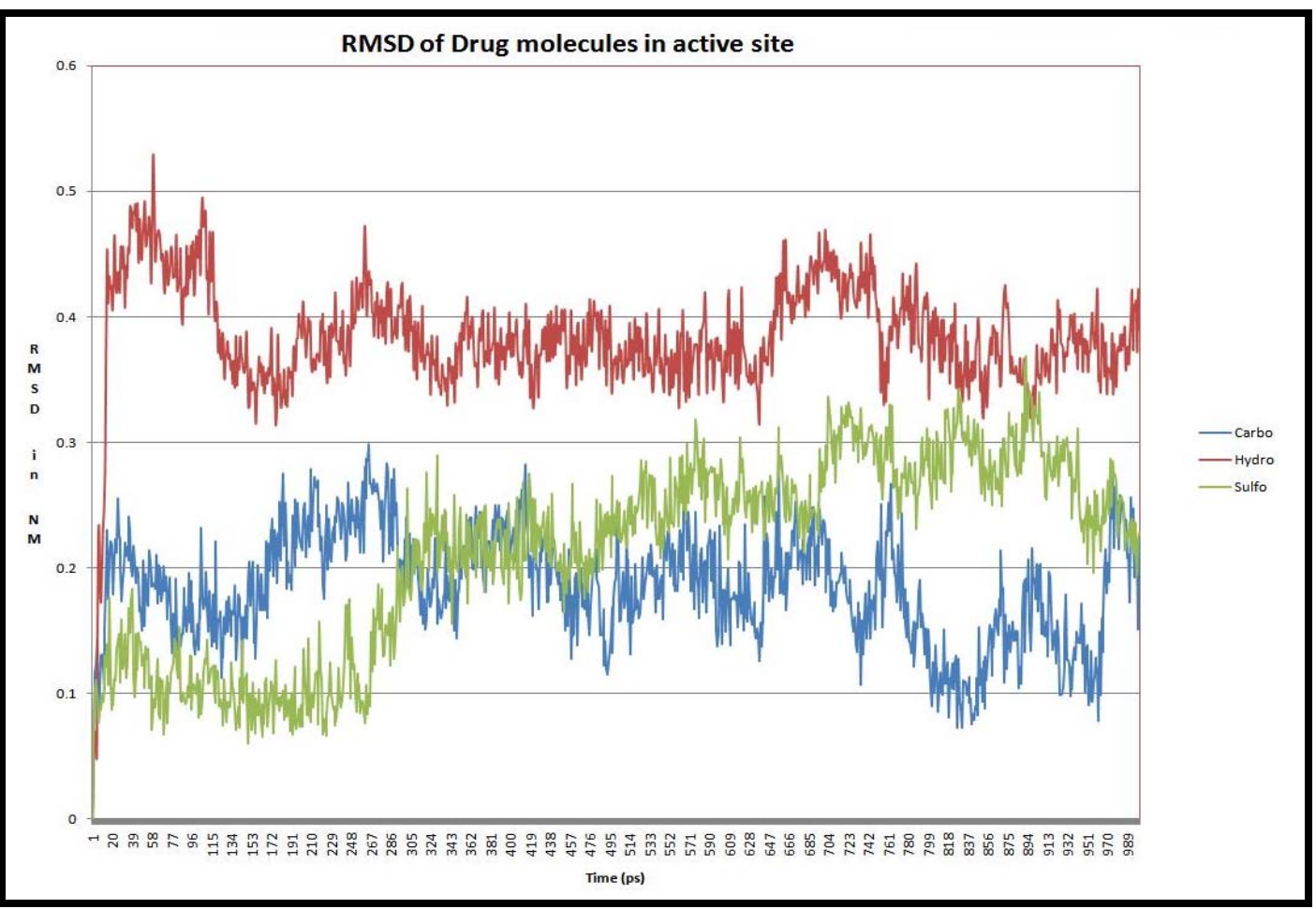

Figure 2: Root mean square deviation between drug molecules and Backbone atoms of MMP9 structure for chosen inhibitors from the three groups.

\section{Acknowledgments:}

We would like to thank Ms Sakshi Rana and for her valuable assistance during this study; we would also like to thank Mr. Sunil Kumar Gupta of bioinformatics center, biotech park Lucknow for his technical assistance on using SYBYL. We would also like to thank Mrs Prachi Srivastava for her assistance and we would specially like to mention Dr. Daya Shankar lal Srivastava for his guidance in initiating the studies.

\section{References:}

[1] K Kessenbrock et al. Cell 141: 52 (2010) [PMID: 20371345]

[2] LJ Mccawley \& LM Matrisian. Curr. Opin. Cell. Biol. 13 : 534 (2001) [PMID: 11544020]

[3] H Li et al. J. Biol. Chem. 284: 4439 (2009) [PMID: 19074147]

[4] ND Rawlings et al. Nucleic Acid. Res. 36: D320 (2008) [PMID 17991683]

[5] G Opdenakker et al. Trends Immunol. 22: 571 (2001) [PMID: 11574282]

[6] M Egeblad \& Z Werb. Nat. Rev. Cancer 2: 161 (2002) [PMID: 11990853]

[7] JM Shipley et al. J. Biol. Chem. 271: 4335 (1996) [PMID: 8626782]
[8] SA Fry et al. Biochemistry 45: 15249 (2006) [PMID: 17176047]

[9] G Bergers et al. Nat. Cell. Biol. 2: 737 (2000) [PMID: 11025665]

[10] MD Sternlicht \& Z Werb. Annu. Rev. Cell. Dev. Biol. 17: 463 (2001) [PMID: 11687497]

[11] LM Coussens et al. Science 295: 2837 (2002) [PMID: 11923519]

[12] A Tochowicz et al. J. Mol. Biol. 371: 989 (2007) [PMID: 17599356]

[13] B Hess et al. J. Chem. Theory. Comput. 4: 435 (2008)

[14] EF Pettersen et al. J. Comput. Chem. 25: 1605 (2004) [PMID: $15264254]$

[15] GM Morris et al. J. Comput. Chem. 30: 2785 (2009) [PMID: 19399780]

[16] SYBYL X Tripos International, 1699 South Hanley Rd., St. Louis, Missouri, 63144, USA.

[17] SP Gupta Chem. Rev. 107: 3042 (2007) [PMID: 17622180]

[18] QX Sang et al. Biochem. Biophys. Res. Commun. 274: 780 (2000) [PMID: 10924354]

[19] RE Babine \& SL Bender. Chem. Rev. 97(5): 1359 (1997) [PMID: 11851455]

Edited by AU Khan

Citation: Tandon \& Sinha, Bioinformation 5(8): 310-314 (2011) License statement: This is an open-access article, which permits unrestricted use, distribution, and reproduction in any medium, for non-commercial purposes, provided the original author and source are credited. 


\section{Supplementary material:}

Table 1: Three Ligand chosen for Molecular Dynamics Study along with their docking scores (Autodock and Flexidock), key H bond residues and RMSD between Autodock and flexidock conformations.

\begin{tabular}{|c|c|c|c|c|c|}
\hline $\begin{array}{c}\text { Ligand Zinc Binding } \\
\text { Group }\end{array}$ & Structure & $\begin{array}{l}\text { Docking Energy } \\
\text { (Autodock) } \\
(\mathrm{Kj} / \mathrm{mol})\end{array}$ & $\begin{array}{l}\text { Docking } \\
\text { Energy } \\
\text { (Flexidock) }\end{array}$ & $\begin{array}{c}\text { RMSD } \\
\text { (Autodock \& } \\
\text { Flexidock) }\end{array}$ & $\begin{array}{l}\mathrm{H} \text { bond residues } \\
\text { and distances }\end{array}$ \\
\hline Carboxylic Group & & -6.09 & -297.64 & $0.093 \AA$ & $\begin{array}{l}\text { Ala } 189(1.824 \AA) \\
\text { Leu } 188(1.810 \AA)\end{array}$ \\
\hline $\begin{array}{l}\text { Sulfonamide } \\
\text { Hydroxamate }\end{array}$ & & -9.04 & -498.68 & $0.533 \AA$ & $\begin{array}{l}\text { Leu } 188(2.325 \AA) \\
\text { Ala } 189(1.856 \AA) \\
\text { Gln } 402(2.265 \AA) \\
\text { His } 276(2.924 \AA) \\
\text { His } 411(1.903 \AA)\end{array}$ \\
\hline Thio ester based ZBG & & -10.02 & -642.45 & $0.078 \AA$ & $\begin{array}{l}\text { Leu } 188(2.292 \AA) \\
\text { Ala } 189(2.217 \AA) \\
\text { Tyr } 420(2.409 \AA) \\
\text { Pro } 421(3.459 \AA) \\
\text { Tyr } 423(1.747 \AA)\end{array}$ \\
\hline
\end{tabular}

